\title{
Linear Time-varying System Control Based On The Inversion Transformation*
}

\author{
M.-S. CHEN $\dagger$ and Y.-R. HUANG $\dagger$
}

Key Words-Linear time-varying systems; continuous time systems: state feedback; controllability grammian; Lyapunov function.

\begin{abstract}
This paper proposes a new state feedback control design for uniformly controllable linear time-varying systems. The new design is based on a nonlinear state transformation that converts the stabilization problem of the system state into a destabilization problem of the transformed state. Such a transformation results in a new control that has two advantages over previous controls. (1) In some of the previous control designs, forecast of future information of the time-varying system matrices over some time interval $[t, t+\Delta]$ is required to calculate the state feedback gain at any time instant $t$, while in the new control design no such forecast is needed. (2) In other previous control designs, consecutive time differentiation on the time-varying system matrices is required, while in the new control design time integration instead of differentiation is applied to the system matrices. The new control can therefore be applied to systems with time-varying parameters that are piecewise continuous in time. (C) 1997 Elsevier Science Ltd.
\end{abstract}

\section{Introduction}

The control design for linear time-varying systems has been a difficult challenge for engineers. The main difficulty arises from the fact that the eigenvalues of a time-varying system matrix cannot he linked with the system's stability property as in the time invariant case (Wu, 1974). Most state feedback control designs that are based on the eigenvalue assignment lack theoretical justification when applied to a time-varying system. Conventional approaches for the time-varying system control have relied on the robust control design, in which the timc-varying system matrix is decomposed into a nominal constant matrix and a time-varying deviation. A robust time-invariant controller (e.g. Petersen, 1985: Bernstein, 1987; Schuilendorf, 1988) is then constructed based on the nominal constant matrix. Although different robustness criteria (e.g. Saberi et al., 1995; Dahleh and Diaz-Bobillo, 1995; Green and Limebeer, 1995) have been proposed, the resultant robust controllers can tolerate the time-varying deviation only up of a certain magnitude. The only exception occurs when the time-varying deviation satisfies a special structural condition called the matching condition. In such a case, a stabilizing controller (Gutman 1979: Corless and Leitmann, 1981) exists for arbitrarily large but bounded time-varying deviations. However, in most cases, the time-varying deviation does not satisfy the matching condition, and stability can be assured only when the size of the time-varying deviation is small in a certain sense (Barmish and Leitmann, 1982)

* Received 31 July 1995; received in final form 1 October 1996. This paper was not presented at any IFAC meeting. This paper was recommended for publication in revised form by Associate Editor E. Kaszkurewicz under the direction of Editor Tamer Başar. Corresponding author Professor E-mail mschen@ccms.ntu.edu.tw.

† Department of Mechanical Engineering, National laiwan University, Taipei. Taiwan, Republic of China. mschen@ccms.ntu.edu.tw.
When information on the time-varying system matrices is known exactly, there are exact control designs that can deal with arbitrarily large time-varying deviations. A particular case is when the system is periodically time-varying. For such a system, stabilizing controls have been developed by Kano and Nishimura (1979), Bittanti et al. (1986), and Calico and Wiesel (1984). When the systems are nonperiodic, such as those subject to nonstationary parametric excitations (Nayfeh and Mook, 1979), the control design becomes more difficult and challenging. Essentially, there are two control design approaches for a nonperiodic system. The first approach is an extension of the eigenvalue assignment control for linear time-invariant systems. Successful examples using this approach can be found in Wolovich (1968), Follinger (1978), and Valasek and Olgac (1993). Their design procedure involves a time-varying transformation of the system into a phase-variable canonical form, and a linear state feedback with a time-varying feedback gain. However, in the calculation of the time-varying feedback gain, the system matrices have to be differentiated with respect to time consecutively up to the order of the system dimension. When information on the system matrices is obtained via sensor measurement, time differentiation of the system matrices will amplify the measurement noise. The higher the order of time differentiation, the more serious the noise-amplification problem. Since the amplified noise can seriously impair the system performance, this approach has been more of theoretical interest than of practical use in most cases. The model matching state feedback control for linear timevarying systems by $\Lambda$ rvanitis and Paraskcvopoulos (1992) also suffers from this disadvantage.

The second approach (e.g. Callier and Desoer, 1992: Rugh. 1993) for nonperiodic system control relies on the Lyapunov stability theorem (Vidyasagar, 1993), where the open-loop controllability grammian is used as a Lyapunov function. This approach has the advantage that no time differentiation is required on the system matrices. However, the calculation of the state feedback gain at any time instant $t$ requires information of the system matrices over some future time interval $[t, t+\Delta]$. Nevertheless, a forecast of how the time-varying system matrices vary over the future time interval $[t, t+\Delta]$ is very difficult in most applications. The same forecast problem also appears in the LQ control (Anderson and Moore. 1989) and in the modified LQ control based on a receding horizon concept (Kwon and Pearson. 1977) for linear time-varying systems. Other approaches for time-varying system control can be found in D'Angelo (1970) and Pinzoni (1989).

The aim of the present paper is to provide a new control design for periodic or nonperiodic systems in order to avoid the disadvantages of the two approaches mentioned above. Specifically, the new control design should require neither a prediction of future information about the system matrices nor time differentiation of the system matrices. The new control design is exact in the sense that it utilizes the exact information of the system matrices to synthesize the controller. 
The notation employed in this paper is summarized as follows. Given a vector $x \in R^{n},\|x\|$ represents the Euclidean norm of the vector. For an arbitrary matrix $A,\|A\|$ denotes the maximum singular value of the matrix: i.e. the induced-two norm of the matrix. The transpose and the inverse of $A$ are denoted by $A^{T}$ and $A^{-1}$, respectively. $\lambda_{i}(A)$ denotes the eigenvalue of $A$, and $\sigma_{i}(A)$ is the singular value of $A$. Given two matrices $A$ and $B, A \geq B$ suggests that $A-B$ is a positive semidefinite matrix $(A-B \geq 0)$. A time-varying matrix $P(t)$ is said to be uniformly positive definite if there is a positive constant $\beta$ such that $P(t) \geq \beta I$ for all $t$.

\section{The inversion transformation}

Consider a multivariable linear time-varying system

$$
\dot{x}=A(t) x+B(t) u, \quad x(0)=x_{0},
$$

where $x(t) \in R^{n}$ is the system state, $u(t) \in R^{n \times m}$ is the control input, and $A(t) \in R^{n \times m}, B(t) \in R^{n \times m}$ are system matrices the elements of which are bounded continuous or piecewise continuous functions of time. The system (1) is assumed to be uniformly controllable, as defined by Chen (1984). The control objective is to find a possible state-dependent feedback gain $K(x, t)$ such that the control input

$$
u=K(x, t) x
$$

stabilizes the system (1) exponentially.

The control to be presented in this paper can be regarded as a modification of the control given by Rugh (1993), which achieves exponential stability of the closed-loop system by linear state feedback with a time-varying feedback gain. However, to implement the control at any time instant $t$, one has to compute the system's controllability grammian defined over some future time interval $[t, t+\Delta]$; in other words, information of the system matrices over a future time interval must be known in advance at any time instant $t$. In the present paper it will be shown that, by introducing a nonlinear inversion transformation, one needs only compute the controllability grammian for the transformed system defined over some past time interval $[t-\Delta, t]$. The resulting control will be a nonlinear state feedback control; however, it has the advantage that no forecast of future information on the system matrices is required.

The key element of the proposed control is the following inversion transformation (Fritz and Courant, 1989) on the state variables of the system (1):

$$
z=\frac{x}{\|x\|^{2}} \text {. }
$$

Note that the norm of the transformed state $z$ is just the inverse of $\|x\|$, i.e.

$$
\|z\|=\frac{1}{\|x\|} .
$$

According to (1) and (2), the governing equation of $z$ can be written as

$$
\begin{aligned}
\dot{z} & =\frac{\dot{x} x^{T} x-2 x x^{T} \dot{x}}{\left(x^{T} x\right)^{2}} \\
& =(A(t) x+B(t) u) \frac{x^{T} x}{\left(x^{T} x\right)^{2}}-\frac{2 x x^{T}}{\left(x^{T} x\right)^{2}}(A(t) x+B(t) u) \\
& =\left(I-2 \frac{x x^{T}}{x^{T} x}\right) A(t) \frac{x}{\|x\|^{2}}+\left(I-2 \frac{x x^{T}}{x^{T} x}\right) B(t) \frac{u}{\|x\|^{2}} \\
& =T(x) A(t) z+T(x) B(t) v,
\end{aligned}
$$

where $v$ is the transformed input

$$
v=\frac{u}{\|x\|^{2}},
$$

and $T(x)$ is a Householder transformation (Chen, 1984)

$$
T(x)=I-2 \frac{x x^{T}}{x^{T} x}
$$

A Householder transformation matrix is symmetric and satisfies the identity (Chen, 1984) $T^{2}(x)=I$. Hence, $T^{-1}(x)$ always exists; namely,

$$
T^{-1}(x)=T(x)
$$

Furthermore, $T(x)$ is uniformly bounded for any nonzero $x$, since

$$
\begin{aligned}
\sigma_{i}(T(x)) & =\sqrt{\lambda_{i}\left(T^{T}(x) T(x)\right)} \\
& =\sqrt{\lambda_{i}(I)}=1, \quad \forall i=1,2, \ldots, n,
\end{aligned}
$$

where $\sigma_{i}(T)$ denotes the singular value of $T$. Note also that the transformed state vector $z$ reverses its direction under the Householder transformation:

$$
\begin{aligned}
T(x) z & =\left(I-2 \frac{x x^{T}}{x^{T} x}\right) \frac{x}{x^{T} x} \\
& =\frac{x}{x^{T} x}-2 \frac{x}{x^{T} x} \\
& =-z .
\end{aligned}
$$

Equivalently,

$$
z=-T^{-1}(x) z
$$

With the above relationship, the transformed state equation (4) becomes

$$
\bar{z}=\bar{A}(x, t) z+\bar{B}(x, t) v
$$

where the transformed system matrices are given by

$$
\begin{aligned}
& \vec{A}(x, t)=-T(x) A(t) T^{-1}(x) \\
& \bar{B}(x, t)=T(x) B(t) .
\end{aligned}
$$

Equation (3) suggests that the inversion transformation (2) has converted the stabilization problem for the system state $x$ into a destabilization problem for the transformed state $z$ in (8). The control objective now becomes finding a transformed input $v$ that can destabilize the transformed system (8) for all nonzero initial conditions $z(0)$.

It is well known (Chen, 1984) that for the uniformly controllable system (1) the controllability grammian, $W(t)$, of $(A(t), B(t))$ is uniformly bounded and uniformly positive definite in the sense that there exist two positive constants $\gamma_{1}$ and $\gamma_{2}$ such that

$$
\gamma_{1} I \leq W(t) \leq \gamma_{2} I, \quad \forall t>0 .
$$

An interesting question arises as to whether the generalized controllabity grammian for the transformed system (8) is also uniformly bounded and uniformly positive definite, where the generalized controllability grammian for the transformed system matrices $(\bar{A}(x, t), B(x, t))$ is defined by

$$
\begin{aligned}
P(t)= & \int_{t-\Delta}^{t} \Psi(t, \tau) \bar{B}(x(\tau), \tau) \\
& \times \bar{B}^{T}(x(\tau), \tau) \Psi^{T}(t, \tau) \mathrm{e}^{\alpha(\tau-t))} \mathrm{d} \tau,
\end{aligned}
$$

in which $\Delta$ and $\alpha \in R^{+}, \Psi(t, \tau) \in R^{n \times n}$ is defined by

$$
\begin{aligned}
\frac{\partial \Psi(t, \tau)}{\partial t} & =\bar{A}(x, t) \Psi(t, \tau) \\
\Psi^{-1}(t, \tau) & =\Psi(\tau, t) \\
\Psi(t, t) & =I
\end{aligned}
$$

and $\bar{A}(x, t)$ is given by $(9)$ and $\bar{B}(x, \tau)$ is given by $(10)$.

The following lemma provides a positive answer to the above question.

Lemma 1 . If the system (1) is uniformly controllable, there 
exists positive constants $\Delta, \beta_{1}$ and $\beta_{2}$ such that the generalized controllability grammian $P(t)$ satisfies

$$
\beta_{1} I \leq P(t) \leq \beta_{2} I, \quad \forall t>0,
$$

Proof. The first part of the proof will establish the lower bound in (15). It can be verified by direct differentiation that any $z(t)$ satisfying the transformed state equation (8) also satisfies the following integral equation:

$$
z(t)=\Psi\left(t, t_{0}\right) z\left(t_{0}\right)+\int_{t_{0}}^{t} \Psi(t, \tau) \bar{B}(x(\tau), \tau) v(\tau) \mathrm{d} \tau,
$$

where $\Psi(t, \tau)$ is defined by (12)-(14).

Since the system (1) is uniformly controllable, it follows from the definition of uniform controllability (Callier and Desoer, 1992) that, given any bounded $x_{0}, x_{\mathrm{f}}$ and any time instant $t$, there exists a $\Delta>0$ and a control $u$ defined for the time interval $[t-\Delta, t]$, such that $u$ drives the system (1) from $x(t-\Delta)=x_{0}$ to $x(t)=x_{f}$. Note that for any nonzero $x$ there is a one-to-one correspondence between $x$ and $z$ in (2), and similarly between $u$ and $u$ in (5). Therefore, it is concluded that, given any bounded and nonzero $z_{1}, z_{\mathrm{f}}$ and any time instant $t$, there exist a $\Delta>0$ and a transformed control $v$ in (8) defined for the time interval $[t-\Delta, t]$, such that $v$ drives the transformed system (8) from $z(t-\Delta)=z_{0}$ to $z(t)=z_{t}$

The above statement together with (16) can now be used to prove the existence of the lower bound in (15). Note that $P(t)$ is, by definition, positive semidefinite. hence, to prove that $P(t)$ is uniformly positive definite, one needs to prove that the matrix $P(t)$ will not become singular eithe asymptutically or at some finite time instant $t=t^{*}$. A contradiction argument will now be used to prove that $P(t)$ will not become singular asymptotically. Assume that $P(t)$ becomes singular asymptotically: in other words, there exist sequences of time instants $t_{i}$ with $\lim _{i \rightarrow \infty} t_{j}=\infty$, positive constants $\alpha_{i}$ with

$$
\lim _{i \rightarrow \infty} \alpha_{i}=0,
$$

and unit vectors $e_{i} \in R^{n}$ such that

$$
e_{i}^{T} P\left(t_{i}\right) e_{i}=\alpha_{i}
$$

Denote $e_{i}^{T} \Phi\left(t_{i}, \tau\right) \bar{B}(\tau)=F_{i}^{T}(\tau)$. Equation (18) can be written

$$
e_{i}^{T} P\left(t_{i}\right) e_{i}=\int_{t_{i}-\Lambda}^{t_{i}} F_{i}^{T}(\tau) F_{i}(\tau) \mathrm{d} \tau=\alpha_{i} .
$$

Since $F_{i}^{T} F_{i}$ is always non-negative, one concludes from (17) that, for all $\tau \in\left[t_{i}-\Delta, t_{i}\right]$

$$
F_{i}^{T}(\tau)=e_{i}^{T} \Psi\left(t_{i}, \tau\right) \bar{B}(\tau) \rightarrow 0, \quad \text { as } \quad i \rightarrow \infty
$$

According to the last statement in the previous paragraph given two bounded and nonzero vectors $e_{i} / 2$, and $\Psi\left(t_{i}-\Delta, t_{i}\right) e_{i} / 2$, there exists a bounded transformed control input $v_{i}$ that drives $z\left(t_{i}-\Delta\right)=\Psi\left(t_{i}-\Delta, t_{i}\right) e_{i} / 2$ to $z\left(t_{i}\right)=e_{i}$. The integral equation (16) (with $t=t_{i}$ and $t_{0}=t_{i}-\Delta$ ) becomes

$$
\frac{e_{i}}{2}=\int_{\left.t_{i}-\right\lrcorner}^{t_{i}} \Psi\left(t_{i}, \tau\right) \bar{B}(\tau) v_{i}(\tau) \mathrm{d} \tau
$$

Multiplying both sides by $e_{i}^{T}$ yields

$$
\begin{aligned}
e_{i}^{T} \frac{e_{i}}{2} & =\int_{t_{i}-s}^{t_{j}} e_{i}^{T} \Psi\left(t_{i}, \tau\right) \bar{B}(x, \tau) v_{i}(\tau) \mathrm{d} \tau \\
& =\int_{t_{i}-\Delta}^{t_{i}} F_{i}^{T}(\tau) v_{i}(\tau) \mathrm{d} \tau .
\end{aligned}
$$

However, according (19), the right-hand side of the equation approaches zero as $i$ approaches infinity, meaning that

$$
\frac{\left\|e_{i}\right\|^{2}}{2}=\frac{1}{2} \rightarrow 0 \quad \text { as } \quad i \rightarrow x
$$

A contradiction is obtained, and this proves that $P(t)$ will not become singular asymptotically.

To prove that $P(t)$ will not become singular at some finite time instant $t=t^{*}$, one can simply repeat the above argument by repladcing $t_{i}$ by $t^{*}$. Finally, one concludes that $P(t)$ is uniformly positive definite in the sense that there exists $\beta_{1}$ such that the left inequality in (15) holds.

The second part of the proof will establish the upper bound in (15). Suppose that the system matrices $A(t)$ and $B(t)$ in (1) are bounded by

$$
\|A(t)\| \leq a, \quad\|B(t)\| \leq b,
$$

where $a$ and $b$ are two constants. It can be verified using (6) and (7) that the transformed system matrices $\bar{A}(x, t)$ and $\bar{B}(x, t)$ in (9) and (10) are also uniformly bounded:

$$
\begin{aligned}
\|\bar{A}(x, t)\| & =\left\|-T(x) A(t) T^{-1}(x)\right\| \\
& \leq\|T(x)\| \cdot\|A(t)\| \cdot\left\|T^{-1}(x)\right\| \leq a, \\
\|\bar{B}(x, t)\| & =\|T(x) B(t)\| \leq\|T(x)\| \cdot\|B(t)\| \leq b .
\end{aligned}
$$

Note that, according to (12) and (13), $\Psi(t, \tau)$ in the definition of $P(t)$ satisfies the following differential equation;

$$
\begin{aligned}
\frac{\partial \Psi(t, \tau)}{\partial \tau} & =\frac{\partial \Psi^{-1}(\tau, t)}{\partial \tau} \\
& =-\Psi^{-1}(\tau, t) \frac{\partial \Psi(\tau, t)}{\partial \tau} \Psi^{-1}(\tau, t) \\
& =-\Psi(t, \tau) \bar{A}(x(\tau), \tau) .
\end{aligned}
$$

Since the above differential equation is linear with bounded coefficients (due to (20)), one concludes that $\Psi(t, \tau)$ can grow, at most, exponentially as a function of $\tau$ when it is integrated from $t=t$ backwards to $\tau=t-\Delta$ with the initial condition $\Psi(t, t)=I$. Therefore, there exists a positive constant $m$ such that

$$
\|\Psi(t, \tau)\| \leq m \quad \forall \tau \in[t-\Delta, t] .
$$

Finally, an upper bound of $P(t)$ can be deduced from (21) and (23):

$$
\begin{aligned}
\|P(t)\| \leq & \int_{t-\Delta}^{t}\|\Psi(t, \tau)\| \cdot\|\bar{B}(x(\tau), \tau)\| \\
& \times\left\|\bar{B}^{T}(x(\tau), \tau)\right\| \cdot\left\|\Psi^{T}(t, \tau)\right\| \mathrm{d} \tau \\
\leq & \int_{t-\Delta}^{t} b^{2} m^{2} \mathrm{~d} \tau=b^{2} m^{2} \Delta .
\end{aligned}
$$

\section{Control design in the transformed domain}

In the previous section, a nonlinear state transformation was proposed for the system (1), and the governing equation for the system in the transformed coordinate was derived. The nonlinear transformation has the characteristics that it converts the stabilization problem of the original system state into a destabilization problem of the transformed state. To destabilize the transformed system (8), the transformed control $v$ can be chosen as

$$
v=\gamma B^{T}(t) T(x) P^{-1}(t) z, \quad \gamma \geq \frac{1}{2} .
$$

In view of (2) and (5), the real control input to the original system (1) turns out to be

$$
u=\gamma B^{T}(t) T(x) P^{-1}(t) x, \quad \gamma \geq \frac{1}{2} .
$$

Note that the control $(25)$ is quite different from the conventional control for linear systems. The $P(t)$ matrix in (11) depends on $x(\tau), \tau \in[t-\Delta, t]$, therefore, the proposed control (25), which contains the inverse of $P(t)$, is actually a nonlinear dynamic state feedback control. The closed-loop system becomes nonlinear, although the open-loop is linear. The following lemma shows that the finite-time escape phenomenon will not take place under the proposed control.

Lemma 2. If the system matrices $A(t)$ and $B(t)$ arc uniformly bounded. and the pair $(A(t), B(t))$ is uniformly controllable, the state variables of the closed-loop system (1) and (25) can grow, at most, exponentially.

Proof. Note first that

$$
\left\|P^{-1}(t)\right\| \leq \frac{1}{\beta_{1}},
$$


according to (15) in Lemma 1, and secondly that

$$
\|T(x)\|=1,
$$

according to (7). The proposed state feedback gain $K(x, t)$ in (25) is therefore bounded by

$$
\|K(x, t)\| \leq \gamma\|B(t)\| \cdot\|T(x)\| \cdot\left\|P^{-1}(t)\right\| \leq \frac{\gamma b}{\beta_{1}},
$$

where $b$ is an upper bound of $\|B(t)\|$. The control input $u(t)$ in (25) is then bounded by

$$
\|u\| \leq \frac{\gamma b}{\beta_{1}} \cdot\|x\| .
$$

By definition of the Euclidean norm of a vector, one has $\|x\|^{2}=x^{T} x$. Taking the time derivative of this identity, and then taking the absolute value, one obtains

$$
\left|\frac{\mathrm{d}}{\mathrm{d} t}\|x\|^{2}\right|=2\|x\|\left|\frac{\mathrm{d}}{\mathrm{d} t}\|x\|\right|=\left|2 x^{T} \dot{x}\right| \leq 2\|x\|\|\dot{x}\|,
$$

where the last inequality results from the Cauchy-Schwarz inequality. Cancelling $2\|x\|$ from the second and last terms from the above equation yields

$$
\left|\frac{\mathrm{d}}{\mathrm{d} t}\|x\|\right| \leq\|\dot{x}\| .
$$

Using (1) and the control bound obtained in (26), it follows from (27) that

$$
\begin{aligned}
\left|\frac{\mathrm{d}}{\mathrm{d} t}\|x\|\right| & \leq\|A(t)\| \cdot\|x\|+\|B(t)\| \cdot\|u\| \\
& \leq a\|x\|+\frac{b^{2} \gamma}{\beta_{1}}\|x\|=\sigma\|x\|,
\end{aligned}
$$

where $a$ is an upper bound of $\|A(t)\|$, and $\sigma=a+b^{2} \gamma / \beta_{1}$. One can therefore write

$$
-\sigma\|x\| \leq \frac{\mathrm{d}}{\mathrm{d} t}\|x\| \leq \sigma\|x\|
$$

Integrating the second inequality gives the desired result

$$
\|x(t)\| \leq\|x(0)\| \mathrm{e}^{\mathrm{rr}},
$$

which suggests that the closed-loop system state $x$ can grow, at most, exponentially.

In the following theorem, it will be shown that the transformed control (24) destabilizes the transformed system (8) exponentially, and, as a result of (3), the control (25) stabilizes the original system (1) exponentially.

Theorem. Consider the system (1) and the state feedback control (25). If the system (1) is uniformly controllable, the closed-loop system is globally exponentially stable.

Proof. Using (12) and (14), it can be verified that $P(t)$ in (11) satisfies the differential equation

where

$$
\begin{aligned}
\frac{\mathrm{d} P(t)}{\mathrm{d} t}= & -D^{T}(t) D(t)+\bar{B}(x, t) \bar{B}^{T}(x, t) \\
& +\bar{A}(x, t) P(t)+P(t) \bar{A}^{T}(x, t)-\alpha P(t),
\end{aligned}
$$

$$
D(t)=\bar{B}^{T}(x(t-\Delta), t-\Delta) \Psi^{T}(t, t-\Delta) \mathrm{e}^{-\alpha \Delta / 2} .
$$

Consequently, $P^{-1}(t)$ satisfies

$$
\begin{aligned}
\frac{\mathrm{d} P^{-1}}{\mathrm{~d} t}= & -P^{-1} \frac{\mathrm{d} p}{\mathrm{~d} t} P^{-1} \\
= & P^{-1} D^{T} D P^{-1}-P^{-1} \bar{B} \bar{B}^{T} P^{-1} \\
& -P^{-1} \bar{A}-\vec{A}^{T} P^{-1}+\alpha P^{-1},
\end{aligned}
$$

where arguments $t$ and $x$ in $\bar{A}, \bar{B}, D$ and $P$ have been suggested for brevity. Choosing a Lyapunov function candidate

$$
V=z^{T} P^{-1} z
$$

it follows from (15) that

$$
\beta_{2}^{-1}\|z\|^{2} \leq V \leq \beta_{1}^{-1}\|z\|^{2} .
$$

Calculating the time derivative of $V$ along the solutions of $(8),(24)$ and (30) yields

$$
\begin{aligned}
\dot{V}= & z^{T}\left(\bar{A}^{T}+\gamma P^{-1} \bar{B} \bar{B}^{T}\right) P^{-1} z \\
& +z^{T} P^{-1}\left(\bar{A}+\gamma \bar{B} \bar{B}^{T} P^{-1}\right) z+z^{T} \dot{P}{ }^{\prime} z \\
= & \alpha z^{T} P^{-1} z+(2 \gamma-1)\left\|\bar{B}^{T} P^{-1} z\right\|^{2}+\left\|D P^{-1} z\right\|^{2} \\
\geq & \alpha z^{T} P{ }^{\prime} z=\alpha V .
\end{aligned}
$$

Therefore, $V$ approaches infinity exponentially and, in view of (31), so does $z$.

Remark 1. Careful observation on (11) and (25) shows that the proposed control has successfully dispensed with the requirement of forecasting future information of the system matrices. Only past and present information of the system matrices, $A(\tau)$ and $B(\tau)$ with $\tau \in[t-\Delta, t]$, is required for the calculation of the proposed state feedback gain at time $t$. Another advantage of the new control is that it can be applied to systems with piecewise continuous time-varying parameters, since no time differentiation is applied to the system matrices.

Remark 2. To examine the effect of the control gain $\gamma(\geq 1 / 2)$, note that when $\bar{B}^{T} P^{-1} z=0$ in (32), the control input $u$ in (25) also becomes zero. Hence, it is conjectured from (32) that when $u \neq 0$ a larger control gain $\gamma$ has the effect of increasing the growth rate of the Lyapunov function $V$, and hence, the effect of increasing the convergence rate of $x$. However, the exact relationship between $\gamma$ and the state convergence rate needs to be investigated further.

Remark 3. The analysis in the Theorem imposes no constraint on the parameter $\Delta$ in the definition of $P(t)$ in (11). However, if $\Delta$ is chosen too small, the integration time interval in $P(t)$ becomes small, and the $P(t)$ matrix will approach singular. This will force the control input $u$ in (25), which contains the inversion of $P(t)$, to become overly large. On the other hand, if $\Delta$ is chosen too large, it would take too much computational time in calculating $P(t)$, as described below.

Before concluding this section, it is advised that the computation of the generalized controllability grammian $P(t)$ in (11) should not be based on the matrix differential equation (28). The reason for this is that in order to run the matrix differential equation (28), one needs to run in parallel another matrix differential equation

$$
\begin{aligned}
\frac{\mathrm{d} \Psi(t, t-\Delta)}{\mathrm{d} t}= & \bar{A}(x(t), t) \Psi(t, t-\Delta) \\
& -\Psi(t, t-\Delta) \bar{A}(x(t-\Delta), t-\Delta)
\end{aligned}
$$

in order to obtain $\Psi(t, t-\Delta)$ appearing in $D(t)$ in (29). This will substantially increase the computational time. Instead, one should compute $P(t)$ directly based on its definition (11) in the following way: integrating the differential equation (22) starting from $\tau=t$ backwards to $\tau=t-\Delta$ with the initial condition $\left.\Psi(t, \tau)\right|_{\tau=t}=I$ gives the matrix value $\Psi(t, \tau)$, $\tau \in[t-\Delta, t]$. Then using these matrix values together with $\bar{B}(x(\tau), \tau)$ to compute the $P(t)$ matrix according to (11). In practical applications, the continuous control $u(t)$ in (25) is approximated by a discretized control $u(k \Delta t)$ with some small sampling time $\Delta t$, and the above computation procedure is done within each sampling interval. Note that the computational work required by the proposed control is no more than that required by the control in Rugh (1993) and Callier and Desoer (1992). 


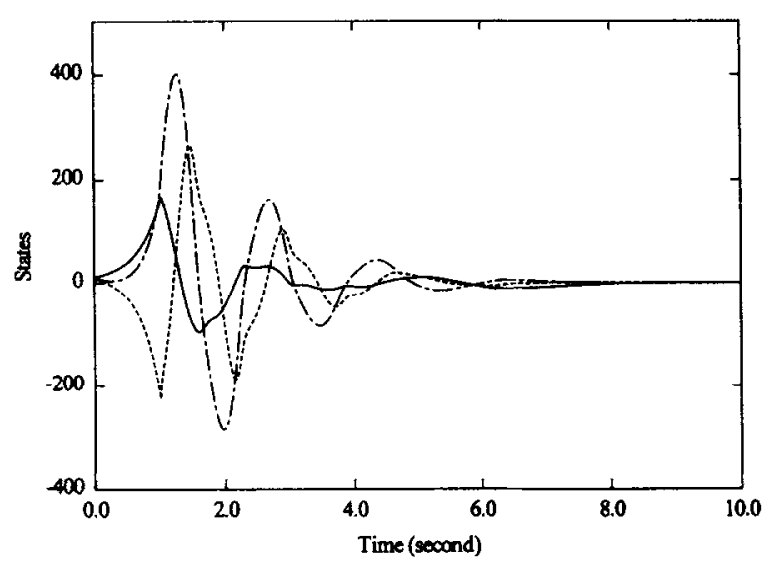

Fig. 1. Time history of system states.

Finally, we present a simulation example.

Example. Consider an open-loop unstable system as in (1) with

$$
\begin{gathered}
A(t)= \\
{\left[\begin{array}{ccc}
-4+7.5 \cos ^{2} \sqrt{t} & 1-7.5 \sin \sqrt{t} \cos \sqrt{t} & 1 \\
-1-7.5 \sin \sqrt{t} \cos \sqrt{t} & -4+7.5 \sin ^{2} \sqrt{t} & 1 \\
0 & -5 \sin \sqrt{t} & -5+5 \sin \sqrt{t}
\end{array}\right]} \\
B(t)=\left[\begin{array}{cc}
1 & 2 \\
3 & 1 \\
1 & 0
\end{array}\right]
\end{gathered}
$$

Note that the system is nonperiodic due to the presence of $\sqrt{t}$. In the simulation, the system runs open loop for the first second with the initial condition $x^{T}(0)=[10,10,10]$. The proposed control (25) is activated at $t=1$ with the following parameters: $\Delta=1, \gamma=0.5$, and $\alpha=0.1$. In the simulation, a discretized control is used to approximate the continuous control with a sampling time $\Delta t=0.01$ second. The performance of the system state and the control input are shown in Figs 1 and 2, respectively; Fig. 1 clearly shows the exponential convergence of the closed-loop system state under the proposed control. Figure 3 shows the minimum singular value of the generalized controllability grammian $P(t)$, which remains uniformly bounded above from zero as predicted by Lemma 1 .

\section{Conclusions}

In this paper, a nonlinear state feedback control design is proposed for linear time-varying systems. Global exponential stability of the controlled system is established if the open-loop system is uniformly controllable. Compared with previous controls for linear time-varying systems, the

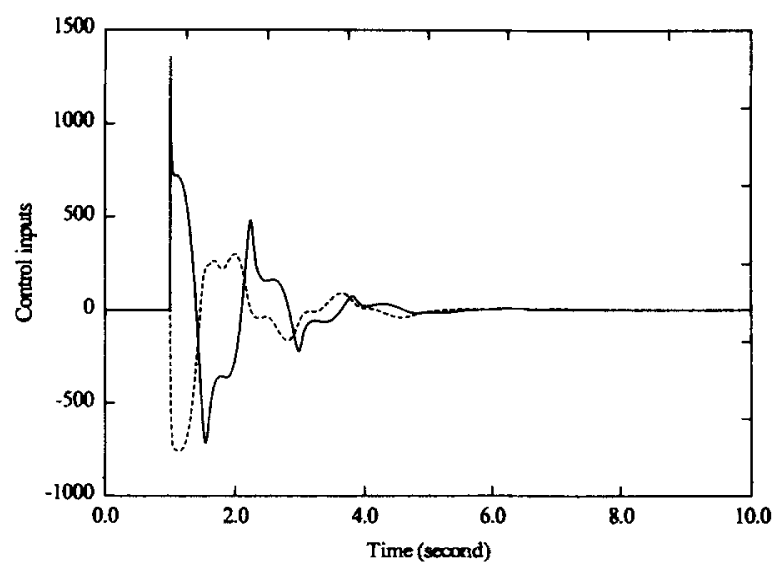

Fig. 2. Time history of control inputs.

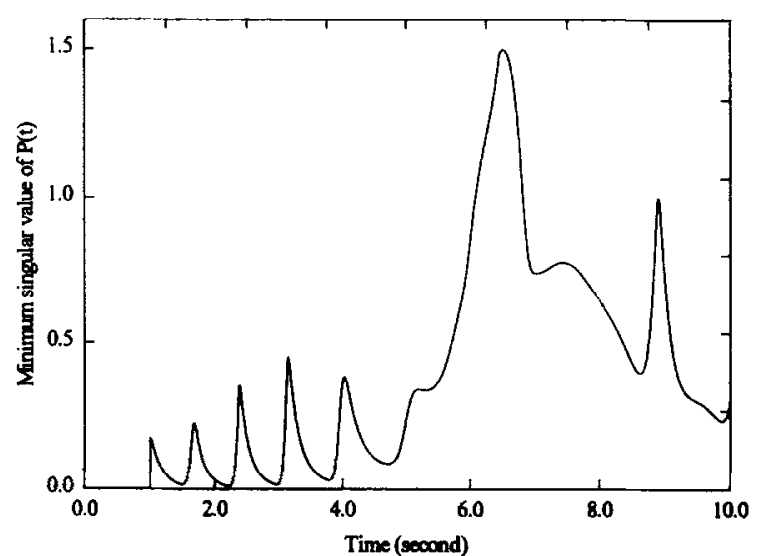

Fig. 3. Minimum singular value of $P(t)$.

proposed control has the advantage that it requires neither forecast of future information of the system matrices nor time derivatives of the system matrices.

Possible future directions of research are as follows. (1) combination of the proposed control with the observer design for linear time-varying system given in Rugh (1993). However, due to the nonlinearity of the proposed control, stability analysis of the observer-based state feedback control is somewhat complicated. (2) Since the proposed control guarantees exponential stability of the closed-loop system, it is expected that the stability result can be retained under small parametric perturbations. In other words, the proposed control should be robust against small parametric uncertainties. To prove this statement rigorously is another subject to be pursued in the future.

Acknowledgement-This work was supported by the National Science Council of the Republic of China under grant number NSC-85-2212-E-002-001.

\section{References}

Anderson, B. D. O. and J. B. Moore (1989). Optimal Control, Linear Quadratic Methods. Prentice-Hall, Englewood Cliffs, NJ.

Arvanitis, K. G. and P. N. Paraskevopoulos (1992). Uniform exact model matching for a class of linear time-varying analytic systems. Syst. Control Lett., 19, 312-323.

Barmish, B. R. and G. Leitmann (1982). On ultimate boundedness control of uncertain systems in the absence of matching assumptions. IEEE Trans. Autom. Control, AC-27, 153-158.

Bernstein, D. S. (1987). Robust static and dynamic output-feedback stabilization: deterministic and stochastic perspectives. IEEE Trans. Autom. Control, AC-32, 1076-1083.

Bittanti, S., P. Colaneri and G. Guardabassi (1986). Analysis of the periodic Lyapunov and Riccati equations via canonical decomposition. SIAM. J. Control Optim., 24, 1138-1149.

Calico, R. A. and W. E. Wiesel (1984). Control of time-periodic systems. J. Guidance, 7, 671-676.

Callier, F. and C. A. Desoer (1992). Linear System Theory. Springer-Verlag, Hong Kong.

Chen, C. T. (1984). Linear System Theory and Design. Holt, Rinehart and Winston, New York.

Corless, M. and G. Leitmann (1981). Continuous state feedback guaranteeing uniform ultimate boundedness for uncertain dynamic systems. IEEE Trans. Autom. Control, AC-26, 1139-1144.

Dahleh, M. A. and I. J. Diaz-Bobillo (1995). Control of Uncertain Systems. Prentice-Hall, Englewood Cliffs. NJ.

D’Angelo, H. (1970). Linear Time-varying Systems: Analysis and Design. Allyn and Bacon, Boston, MA.

Follinger, $O$. (1978). Design of time-varying system by pole assignment. Regelungstechink, 26, 85-95. 
Fritz, J. and R. Courant (1989). Introduction to Calculus and Analysis, Vol. II. Wiley-Interscience, New York.

Green, M. and D. J. N. Limebeer (1995). Linear Robust Control. Prentice-Hall, Englewood Cliffs, NJ.

Gutman, S. (1979). Uncertain dynamic systems-a Lyapunov min-max approach. IEEE Trans. Autom. Control, AC-24, 437 443 .

Kano, H. and T. Nishimura (1979). Periodic solutions of matrix Riccati equations with detectability and stabilizability. Int. J. Control, 29, 471-487.

Kwon, W. H. and A. E. Pearson (1977). A modified quadratic cost problem and feedback stabilization of a linear system. IEEE Trans. Autom. Control, AC-22, 838-842.

Nayfeh, A. H. and D. T. Mook (1979). Nonlinear Oscillations. Wiley-Interscience, New York.

Petersen, I. R. (1985). A Riccati equation approach to the design of stabilizing controllers and observers for a class of uncertain linear systems. IEEE Trans. Autom. Control, AC-30, 904-907.
Pinzoni, S. (1989). Stabilization and control of linear time-varying systems. PhD Thesis, Arizona State University.

Rugh, W. J. (1993). Linear System Theory. Prentice-Hall, Englewood Cliffs, NJ.

Saberi, A., P. Sannuti and B. M. Chen (1995). $\mathrm{H}_{2}$ Optimal Control. Prentice-Hall, Englewood Cliffs, NJ.

Schmitendorf, W. E. (1988). Designing stabilizing controllers for uncertain systems using the Riccati equation approach. IEEE Trans. Autom. Control, AC-33, 376-379.

Valasek, M. and N. Olgac (1993). Generalization of Ackermenn's formula for linear MIMO time invariant and time varying systems. In Proc. 32nd IEEE Conf. on Decision and Control, San Antonio, TX, pp. 827-831.

Vidyasagar, M. (1993). Nonlinear Systems Analysis. PrenticeHall, Englewood Cliffs, NJ.

Wolovich, W. A. (1968). On the stabilization of controllable systems. IEEE Trans. Autom. Control, AC-13, 569-572.

Wu, M. Y. (1974). A note on stability of linear time-varying systems. IEEE Trans. Autom. Control, AC-19, 162-163. 